\title{
Características de mulheres obesas assistidas pelo Núcleo de Apoio à Saúde da Família
}

\author{
Characteristics of obese women receiving care from a Family Health Support Unit \\ Características de mujeres obesas asistidas por el Núcleo de Apoyo a la Salud de la Familia
}

\author{
Sabrina Bezerra da Silval; Rafaella Queiroga Souto"; Fábia Alexandra Pottes Alves"'; Tibério Gambarra Morais"'; \\ Gleicy Karine Nascimento de Araújov; Mikellayne Barbosa Honoratov".
}

\begin{abstract}
RESUMO
Objetivo: descrever a eficácia do grupo de reeducação alimentar e controle de peso do Núcleo de Apoio à Saúde da Família (NASF) no município de Santa Luzia-PB. Método: trata-se de um ensaio comunitário, realizado no período de abril a setembro de 2015 . As participantes foram avaliadas por meio de uma consulta e avaliação nutricional antes e depois da realização das atividades educativas. A população é composta por 15 pacientes do sexo feminino, com Índice de Massa Corporal (IMC) acima de 30Kg/m². Temas como hábitos saudáveis e práticas de atividades físicas foram discutidos em palestras expositivas e rodas de conversas. Resultado: verificou-se redução significativa no IMC das pacientes ( $p=0,000$ ), de $36,22 \mathrm{Kg} / \mathrm{m}^{2}$ (antes da intervenção) para $32,94 \mathrm{Kg} / \mathrm{m}^{2}($ depois), evidenciando a importância do grupo na melhoria da qualidade de vida dos usuários. Conclusão: a redução do IMC foi satisfatória, reforçando a relevância da equipe do NASF e manutenção do grupo.
\end{abstract}

Descritores: Obesidade; saúde da família; índice de massa corporal; atenção primária à saúde.

\section{ABSTRACT}

Objective: to describe the efficacy of the eating re-education and weight control group of the Family Health Support Center (FHSC) in the city of Santa Luzia, Paraíba. Method: in this community trial, conducted from April to September 2015, participants were evaluated by nutritional consultation and nutritional assessment before and after the educational activities. The population comprised 15 female patients, with body mass index (BMI) above $30 \mathrm{~kg} / \mathrm{m}^{2}$. Issues such as healthy habits and physical activities were discussed in lectures and conversation circles. Results: significant reduction was observed in participants' BMI $(p=0.000)$, from $36.22 \mathrm{~kg} / \mathrm{m}^{2}(\mathrm{before}$ the intervention) to $32.94 \mathrm{~kg} / \mathrm{m}^{2}$ (afterwards), evidencing the importance of the group in improving the quality of users' lives. Conclusion: $\mathrm{BMI}$ reduction was satisfactory, underlining the importance of the FHSC team and of maintaining the group. Descriptors: Obesity; family health; body mass index; primary health care.

\section{RESUMEN}

Objetivo: describir la eficacia del grupo de reeducación alimentaria y control de peso del Núcleo de Apoyo a la Salud de la Familia (NASF) en el municipio de Santa Luzia-Paraíba. Método: se trata de un ensayo comunitario, realizado en el período de abril a septiembre de 2015. Las participantes fueron evaluadas por medio de consulta y evaluación nutricional antes y después de la realización de las actividades educativas. La población se compone de 15 pacientes del sexo femenino, con Índice de Masa Corporal (IMC) superior a $30 \mathrm{Kg} / \mathrm{m}^{2}$. Los temas como hábitos saludables y prácticas de actividades físicas se discutieron en charlas expositivas y ruedas de conversación. Resultados: se observó una reducción significativa en el IMC de las pacientes $(p=0,000)$, de $36,22 \mathrm{Kg} / \mathrm{m}^{2}(\mathrm{antes}$ de la intervención) a $32,94 \mathrm{Kg} / \mathrm{m}^{2}$ (después), evidenciando la importancia del grupo en la mejora de la calidad de la vida de los usuarios. Conclusión: la reducción del IMC fue satisfactoria, reforzando la relevancia del equipo del NASF y el mantenimiento del grupo. Descriptores: Obesidad; salud de la familia; indice de masa corporal; atención primaria de salud.

\section{INTRODUÇÃO}

A transição demográfica e epidemiológica vivenciada atualmente demonstra uma redução das doenças infecciosas e aumento de Doenças Crônicas Não Transmissíveis (DCNT), assim como a transição nutricional que reflete na diminuição da desnutrição e aumento do excesso de peso ${ }^{1}$. Em 2012, cerca de 38 milhões de pessoas no mundo veio a óbito devido às consequências DCNT, sendo a obesidade um dos maiores fatores de risco para adoecimento ${ }^{2}$.

A partir disso, foi desenvolvida uma linha de cuidados na atenção básica para excesso de peso e outros fatores de risco associados ao sobrepeso e a obesidade, que abrange desde o acolhimento até o atendimento em serviços especializados ${ }^{1}$.

'Nutricionista. Mestre. Diretora geral do Hospital e Maternidade Sinhá Carneiro. Paraíba, Brasil. E-mail: sabrinabs@gmail.com "Enfermeira. Pós doutora. Professora Adjunta, Universidade Federal da Paraíba. Brasil. E-mail: rafaellaqueiroga7@gmail.com '"Enfermeira. Doutora. Professora Adjunta, Universidade Federal de Pernambuco. Brasil. E-mail: fabia.alexandra@terra.com.br IVFisioterapeuta. Diretora geral do Hospital e Maternidade Sinhá Carneiro. Paraíba, Brasil. E-mail: tiberiogm@gmail.com vEnfermeira. Universidade Federal de Pernambuco. Brasil. E-mail: gleicy.kna@hotmail.com

viEnfermeira. Universidade Federal de Pernambuco. Brasil. E-mail: mikellaynebhonorato@gmail.com 
A pessoa que apresenta sobrepeso pode ser direcionada pela equipe das unidades básicas de saúde (UBS) a um polo do Programa Academia da Saúde para realização de atividades físicas e ao Núcleo de Apoio à Saúde da Família (NASF) para receber orientações sobre alimentação saudável e balanceada. A UBS representa um dos primeiros contatos do sistema de saúde, coordenando a assistência dentro do próprio sistema².

A criação dos NASF objetivou ampliar as ações da atenção básica, apoiando a inserção da ESF na rede de serviços. Acredita-se que, sob a orientação desses profissionais, mediante mudanças no estilo de vida, os casos de sobrepeso e obesidade poderão ser revertidos e prevenidos, impedindo ou retardando a manifestação de doenças crônicas não transmissíveis associadas ao excesso de peso ${ }^{3}$.

As intervenções para reverter o excesso de peso da população necessitam articular as diversas visões da sociedade sobre alimentação, atividade física, corpo e saúde. A UBS, além de acolher e tratar o indivíduo com excesso de peso, deve ser a ordenadora do cuidado e centro de comunicação entre os demais pontos da Rede de Atenção à Saúde (RAS), garantindo o cuidado integral, buscando a interdisciplinaridade e intersetorialidade ${ }^{1}$.

Outro caminho no combate ao excesso de peso tem sido a aplicação do plano de ações estratégicas para o enfrentamento das DCNT, que tem como uma das finalidades deter o crescimento da proporção de adultos brasileiros com excesso de peso ou com obesidade até $2022^{4}$.

Diante desse contexto, o objetivo do presente estudo foi descrever a comparação do Índice de Massa Corporal (IMC) das pacientes assistidas pelo NASF, no município de Santa Luzia- PB, antes e depois da sua participação no grupo de reeducação alimentar e controle de peso.

\section{REVISÃO DE LITERATURA}

No Brasil, as prevalências desses dados em adultos aumentaram desde as pesquisas realizadas na década de 1970. A obesidade no país cresceu $60 \%$ em 10 anos, passando de 11,8\% em 2006 para 18,9\% em 2016

O Instituto Brasileiro de Geografia e Estatística (IBGE) segue os parâmetros da Organização Mundial da Saúde (OMS) na classificação de sobrepeso (IMC superior a $25 \%$ ) e obesidade (IMC superior a $30 \%)^{6}$.

Em relação ao excesso de peso, foi observado que em 2013, 33,2\% da população adulta apresentava sobrepeso e $17,5 \%$ obesidade. A prevalência de sobrepeso e de obesidade aumentou significativamente em ambos os sexos, em todos os níveis de escolaridade e em todas as regiões, sendo esta considerada uma epidemia no Brasil ${ }^{7-9}$.

No entanto, as mulheres, por sua vez, representam um grupo de vulnerabilidade ao sobrepeso e obesidade, pela transição das fases vivenciadas em todo o ciclo vital desde a menarca, período gravídico-puerperal e climatério $^{10,11}$. Apesar disso, estudos apontam o sexo feminino com maior predisposição a diminuir peso corporal e, principalmente por influência da sociedade atual, na percepção da imagem corporal, buscando adequar-se aos corpos magros e ideais que the são impostos ${ }^{12}$. Em sua maioria, as mulheres com obesidade ou sobrepeso relatam não estar satisfeitas com a sua imagem corporal ${ }^{13}$.

Os valores de IMC entre 25 e 30 são responsáveis pela maior parte do impacto do sobrepeso sobre certas comorbidades associadas à obesidade. Em uma pesquisa, identificou-se que cerca de $36,6 \%$ dos homens e $62,9 \%$ das mulheres obesas apresentavam entre 2 ou 3 comorbidades. Destacou-se a população feminina em que $80 \%$ apresentavam Diabetes Mellitus (DM) e que poderiam, teoricamente, prevenir a doença se tivessem um IMC menor ou igual a $25^{14}$.

Apesar da mudança na busca por hábitos saudáveis da população brasileira, os níveis de obesidade e sobrepeso ainda são preocupantes no país. Resultados apontam que um em cada cinco brasileiros são obesos e que $54 \%$ da população das capitais brasileiras estão com excesso de peso; a prevalência da doença passou de 11,8\% em 2006 para 18,9\% em $2016^{15}$. Esse crescimento da obesidade pode ter influenciado no aumento da prevalência de comorbidades, destacando-se a hipertensão e diabetes ${ }^{16}$.

\section{Metodologia}

Trata-se de um ensaio comunitário com abordagem quantitativa. É considerado um ensaio aquele estudo onde os participantes podem ser avaliados após realizações de intervenções ${ }^{17}$. O estudo foi desenvolvido no NASF do município de Santa Luzia, no período de abril a setembro de 2015.

Para a seleção da amostra, foram encaminhados pela ESF ao NASF todos os usuários portando as seguintes características: IMC acima de $30 \mathrm{Kg} / \mathrm{m}^{2}$ e a condição de convidado a participar do grupo de reeducação alimentar e controle de peso. Foram incluídos 22 participantes, no entanto, sete desistiram por incompatibilidade de horário, resultando uma amostra final de 15 integrantes.

A coleta de dados foi realizada por meio de uma consulta e avaliação nutricional antes e depois da realização das atividades educativas. Foram coletadas informações socioeconômicas (idade, sexo, profissão, estado civil, moradia e renda) com objetivo de caracterizar a população.

Para a realização da avaliação nutricional, foram verificados o peso e a altura dos voluntários. A obtenção do peso foi medida em quilogramas, mediante o uso de balança Filizola do tipo plataforma de 0,1 kg, estando o paciente sem adornos e sem sapatos. Para a verificação da altura, medida em centímetros, foi utilizada uma régua antropométrica fixada na própria balança com precisão de $0,1 \mathrm{~cm}$, e para este fim, foi solicitado que o paciente se mantivesse ereto e com as pernas e calcanhares juntos, os braços ao longo do corpo e olhar para o horizonte, sem hiperextensão da coluna. 
Para o cálculo do IMC, foi utilizado a fórmula de (peso em $\mathrm{kg}$ dividido pelo quadrado da altura em metros) para avaliação do estado nutricional de acordo com a OMS.

Os dados coletados foram digitados no programa Microsoft Excel versão 97-2003 e, importados para o SPSS versão 20 , onde foram analisados por meio de estatística descritiva e inferencial.

Para avaliar o impacto do grupo de reeducação alimentar no estado nutricional dos participantes, ou seja, se houve uma redução significativa do IMC, foi realizado o teste de Student para amostras pareadas, já que os mesmos indivíduos foram avaliados em dois momentos distintos. Para aplicação do teste, supõe-se que as amostras seguem uma distribuição normal e para tal foram realizados os testes de Kolmogorov-Smirnov e Shapiro-Wilk.

Adicionalmente, foram construídos intervalos de confiança para a média amostral, considerando as variáveis quantitativas contínuas. Para todos os testes foi estabelecido um nível de confiança de $95 \%$.

Aconteceram encontros semanais entre abril a setembro de 2015, totalizando 17 encontros, com duração de uma hora cada, objetivando trabalhar práticas alimentares e hábitos saudáveis. A partir disso, o foco das intervenções foi para uma conscientização sobre alimentação saudável e desenvolvimento de atividade física, tendo sido discutidos temas sobre motivação e percepção de si, com dinâmicas e terapia de grupo.

Este estudo foi aprovado pelo Comitê de Ética em Pesquisa do Centro de Ciência da Saúde (CCS) do Hospital Universitário Lauro Wanderley da Universidade Federal da Paraíba (UFPB), através do $n$ o de protocolo da CCAE 41786615.9.0000.5183. Foi seguida a Resolução no 466 de 12 de dezembro de 2012, na qual o Conselho Nacional de Saúde (CNS) aprovou as diretrizes e regulamentação de pesquisa que envolve seres humanos, permitindo assegurar os direitos e deveres que dizem respeito à comunidade científica, aos sujeitos da pesquisa e ao Estado.

Antes das coletas dos dados foi explicado aos usuários o objetivo da pesquisa, apresentado o Termo de Consentimento Livre e Esclarecido (TCLE), com destaque para a liberdade de participação e a garantia do anonimato.

\section{RESUltAdos e Discussão}

A população é composta por 15 mulheres, com IMC acima de $30 \mathrm{Kg} / \mathrm{m}^{2}$. Mais da metade - 8(53,34\%) das entrevistadas apresentam idade entre 43 e 55 anos, enquanto que apenas $2(13 \%)$ relataram idade superior a 55 anos. A maioria das mulheres era casada - 11 (73,3\%), e tinha casa própria - 13 (86,6\%). Considerando a distribuição de frequências para a variável renda, verificou-se que $7(46,7 \%)$ pacientes recebem 3 salários mínimos, enquanto que apenas $4(26,7 \%)$ recebem menos de 3 salários, conforme descritos na tabela 1.
TABELA 1: Características socioeconômicas dos participantes do estudo. Santa Luzia, PB,2015.

\begin{tabular}{|c|c|c|}
\hline Características & $f$ & $\%$ \\
\hline \multicolumn{3}{|l|}{ Faixa etária } \\
\hline 19 a 42 anos & 5 & 33,33 \\
\hline 43 a 55 anos & 8 & 53,34 \\
\hline 56 a 64 anos & 2 & 13,33 \\
\hline \multicolumn{3}{|l|}{ Renda } \\
\hline 1 a 2 salários $\left.{ }^{*}\right)$ & 4 & 26,68 \\
\hline 3 a 4 & 10 & 66,66 \\
\hline 5 ou mais & 1 & 6,66 \\
\hline \multicolumn{3}{|l|}{ Profissão } \\
\hline Dolar & 4 & 26,68 \\
\hline Aposentada & 1 & 6,66 \\
\hline Funcionária pública & 2 & 13,33 \\
\hline Vendedora & 2 & 13,33 \\
\hline Outros & 6 & 40,00 \\
\hline \multicolumn{3}{|l|}{ Estado civil } \\
\hline Solteira & 3 & 20,00 \\
\hline Casada & 11 & 73,34 \\
\hline Viúva & 1 & 6,66 \\
\hline \multicolumn{3}{|l|}{ Moradia } \\
\hline Própria & 13 & 86,67 \\
\hline Alugada & 2 & 13,33 \\
\hline
\end{tabular}

${ }^{(*)}$ valor do salário mínimo durante o período da pesquisa $=\mathrm{R} \$ 788,00$.

É nas camadas mais jovens da população que as medidas voltadas para as mudanças de comportamento podem propiciar maior efeito, assim, a faixa etária entre 10 a 19 anos, é considerada a mais efetiva para a concentração de esforços direcionados ao combate ao sobrepeso e à obesidade ${ }^{18}$.

Em relação ao fator renda, em nações em desenvolvimento, como o Brasil, existe uma relação da obesidade com o maior nível socioeconômico. Esse fato se deve pela existência de uma maior disponibilidade de alimentos com maior densidade de energia e pela pouca prática de atividade física nesse perfil socioeconômico ${ }^{2}$.

A condição de solteira, separada ou viúva, ou seja, de não estar engajada em uma relação mais estável, parece proteger a mulher dos distúrbios nutricionais, em comparação às mulheres casadas ou em união conjugal, que apresentaram um risco maior de serem obesas ${ }^{19}$.

No que diz respeito às intervenções, após a apresentação do grupo de reeducação alimentar e da equipe do NASF, ocorreram três encontros em que foram abordados temas como percepção sobre si, tabus alimentares e atividade física diária, através de palestras expositivas, orientações e dinâmicas de grupo.

Nos encontros subsequentes, foram discutidas as dificuldades encontradas, por meio de uma roda de conversa, além de palestras expositivas e dinâmicas. Os temas debatidos foram: imposição de limites pessoais; importância da postura e sua influência na saúde; preparo dos alimentos e receitas saudáveis; favorecimento da autoestima; construção de comportamentos saudáveis.

Durante as oficinas, foram confeccionados cartazes, nos quais os participantes expressavam os seus sentimentos e suas expectativas sobre o desenvolvimento do grupo. A maioria apresentava angústia sobre o 
excesso de peso e esperava redução de peso e das taxas de exames bioquímicos. As atividades de orientação sofreram adaptações frente às reações do grupo, tornando possível a participação de forma dinâmica e consciente.

Em relação aos resultados descritivos e inferenciais da comparação do IMC inicial e final dos participantes, destaca-se que houve redução do IMC final, segundo a Tabela 2.

TABELA 2: Comparação do IMC dos participantes antes e depois das atividades educativas. Santa Luzia/ PB, 2015

\begin{tabular}{lcccc}
\hline \multicolumn{1}{c}{ Estatística } & IMC inicial & IMC final & $\mathbf{t}^{(*)}$ & $\mathbf{p}^{(* *}$ \\
\hline Média & 36,22 & 32,94 & & \\
Mediana & 37,19 & 33,80 & & \\
Desvio padrão & 4,31 & 4,01 & 9,238 & 0.000 \\
Mínimo & 30,00 & 26,49 & & \\
Máximo & 45,39 & 40,75 & & \\
\hline
\end{tabular}

${ }^{(*)}$ teste $t$ de Student

${ }^{(* *)}$ valor de significância do teste

Nota-se que o IMC médio antes da participação no grupo foi de $36,22 \mathrm{Kg} / \mathrm{m}^{2}$ com desvio padrão de $4,31 \mathrm{Kg} /$ $\mathrm{m}^{2}$. Além disso, o IMC mediano alcançou $37,19 \mathrm{Kg} / \mathrm{m}^{2}$, indicando que $50 \%$ da população de interesse apresenta IMC superior ou igual a este valor. Desse modo, é possível constatou-se que mais da metade da população estava inicialmente, classificada em situação de obesidade grau II ou obesidade grau III segundo a classificação da OMS.

Segundo a classificação do IMC das pacientes, considerando os dois períodos em que foram realizadas as medições, verificou-se, que antes da participação no grupo de reeducação alimentar, $7(46,6 \%)$ das pacientes foram classificadas pelo critério da OMS como portadoras de obesidade grave e $6(40,0 \%)$ com obesidade moderada. Após a reeducação dos hábitos alimentares através do grupo, verificou-se uma redução substancial dos casos de obesidade, passando, para 5(33,3\%) portadoras de obesidade grave $4(26,6 \%)$ clientes com obesidade moderada. Além disso, observou-se que $5(33,33 \%)$ pacientes assistidas deixaram de ser classificadas com algum tipo de obesidade, comprovando a efetividade do trabalho de grupo na melhoria da qualidade de vida das pacientes.

Após a participação no grupo de reeducação, nota-se uma redução no IMC dos pacientes quando comparado ao IMC inicial, passando de $36,22 \mathrm{Kg} / \mathrm{m}^{2}$ para 32,94 $\mathrm{Kg} / \mathrm{m}^{2}$. O IMC mediano final foi de $33,80 \mathrm{Kg} / \mathrm{m}^{2}$, enquanto que o mínimo e máximo, foram, respectivamente, 26,49 $\mathrm{Kg} / \mathrm{m}^{2}$ e $40,75 \mathrm{Kg} / \mathrm{m}^{2}$, valores inferiores aqueles obtidos na primeira medição, conforme Tabela 2.

Com base no resultado do teste $t$, verifica-se, com um nível de confiança de $95 \%$, que houve redução significativa $(p=0,000)$, sob o ponto de vista estatístico, no IMC médio das pacientes assistidas pelo NASF. Sendo evidenciada a importância das práticas educativas do grupo de reeducação alimentar para mudanças dos hábitos alimentares dos pacientes investigados, e, consequentemente, implicando a melhoria da qualidade de vida. A efetividade da educação em saúde deve-se ao fato de se tratar de um processo contínuo, que objetiva a promoção da saúde, a partir da participação dos usuários ${ }^{20}$.

Dessa forma, as práticas educativas coletivas permitem o conhecimento das causas e consequências da obesidade, contribuindo para a participação ativa dos usuários de serviços de atenção básica. Portanto, a equipe de saúde deve atuar de forma significativa na educação em saúde visando à prevenção e ao controle do excesso de peso e suas consequências.

Com o aumento da obesidade em nível mundial, as mudanças nos estilos de vida e hábitos saudáveis são temáticas que vêm sendo trabalhadas em todas as faixas etárias no âmbito da saúde e em diversos ambientes como em empresas, escolas e creches. Tais práticas têm alcançado grandes resultados no que diz respeito à saúde física e mental dos praticantes e, consequentemente, na qualidade de vida ${ }^{21}$.

Observou-se, em curto prazo, que houve uma boa adaptação em relação à necessidade de mudança de estilo de vida das participantes, como práticas de atividades físicas e aquisição de hábitos alimentares.

Essas ações tendem a apresentar resultados satisfatórios, podendo promover uma melhora significativa da qualidade de vida e redução nos índices de obesidade. $\mathrm{O}$ acompanhamento nutricional pode servir como ferramenta para a redução do peso corporal e consequente redução dos riscos associados à obesidade e melhora da qualidade de vida ${ }^{22}$.

Um estudo que mais de $80 \%$ das mulheres obesas, em qualquer grau, mostraram-se insatisfeitas com a sua imagem corporal. Apesar do elevado índice de insatisfação, somente $30 \%$ das mulheres com obesidade realizaram alguma ação para reduzir o peso, tais como dietas, atividades físicas ou uso de medicamentos ${ }^{23}$. A percepção do corpo obeso, na mulher, pode ocasionar um pensamento de perda do mundo anterior, banalização da vida cotidiana e exclusão de perspectivas de um futuro melhor ${ }^{24}$.

Para maior redução da perda do IMC, o grupo deveria ser acompanhado a longo prazo, principalmente para obtenção do peso ideal. Diante disso, as atividades de prevenção em grupo são úteis, salientando o fato de que as clientes demonstraram uma boa compreensão da forma como as orientações foram conduzidas, valorizando as informações que lhes foram emitidas.

\section{CONCLUSÃo}

Os resultados mostram uma redução significativa do IMC após a participação das mulheres no grupo do NASF, reforçando a necessidade da manutenção do grupo de reeducação alimentar e a importância da participação da equipe no NASF na atenção primária à saúde. 
Os resultados das intervenções foram satisfatórios por apresentarem alterações positivas nos estilos de vida das clientes, melhorando o bem-estar em geral. Todas demonstraram interesse pelas temáticas abordadas, participando dos relatos sobre as suas angústias e dificuldades individuais e as superações conquistadas nas atividades cotidianas.

As ações de prevenção e promoção da saúde voltadas para portadores de obesidade são salutares, principalmente pelo fato de que a população, geralmente, não dispõe de tratamento imediato e acompanhamento minucioso, comparando os dados registrados no início e após as ações educativas desenvolvidas.

Os profissionais de saúde têm grandes desafios para trabalhar com essa demanda, por isso é importante valorizar os grupos de intervenções. Eles facilitam o alcance da eficácia da promoção e favorecendo dos agravos à saúde, promovendo a motivação e o bem-estar dos usuários com obesidade.

Recomenda-se que este estudo seja realizado em outros contextos, permitindo que essa seja uma alternativa de intervenção implementada pela equipe de saúde para a redução do IMC. Além disto, estudos longitudinais com maiores populações devem ser conduzidos, superando, assim, as limitações do presente estudo.

\section{REFERÊNCIAS}

1. Ministério da Saúde (Br). Estratégias para o cuidado da pessoa com doença crônica: obesidade. Brasília (DF): Editora MS; 2014. 2. Gonçalves RMDA, Lancman S, Sznelwar LI, Cordone NG, Barros JDO. Estudo do trabalho em Núcleos de Apoio à Saúde da Família (NASF), São Paulo, Brasil. Rev. bras. saúde ocup. 2015; 40(131): 59-74.

3. Pedraza DF, Menezes TN, Costa GMC. Ações de alimentação e nutrição na estratégia saúde da família: estrutura e processo de trabalho. Rev. enferm. UERJ. 2016; 24(4): e15848.

4. World Health Organization. Obesity preventing and overweight. Report of a WHO Consultation on Obesity. Geneva (SWI): WHO; 2015.

5. Brasil (Br). Ministério da Saúde. Vigitel Brasil. Hábitos dos brasileiros impactam no crescimento da obesidade e aumenta prevalência de diabetes e hipertensão. 2016. [Acesso em 15 agosto 2018]. Disponível em: http://portalarquivos.saude.gov. br/images/pdf/2017/abril/17/Vigitel.pdf

6. World Health Organization. Obesity preventing and overweight. Report of a WHO Consultation on Obesity. Geneva (SWI): WHO; 2015. 7. Campos DA, Vieira M, Pires ROM. Avaliação do processo de trabalho dos nutricionistas dos Núcleos de Apoio à Saúde da Família do Meio Oeste de Santa Catarina. Saúde transform. soc. 2017; 8(1): 84-97.
8. Santos MC, Frauches MB, Rodrigues SM, Fernandes ET. Processo de trabalho do Núcleo de apoio à Saúde da Família (NASF): importância da qualificação profissional. Saúde transform. soc. 2017; 8(2): 60-69.

9. Malta DC, Santos MAS, Andrade SSCDA, Oliveira TP, Stopa SR, Oliveira MMD, Jaime P. Tendência temporal dos indicadores de excesso de peso em adultos nas capitais brasileiras, 2006-2013. Ciênc. saúde coletiva (Online). 2016; 21(4): 1061-69.

10. Ferreira RAB, Benicio MHDA. Obesidade em mulheres brasileiras: associação com paridade e nível socioeconômico. Rev. Panam. Salud Publica. 2015; 37(4/5): 337-42.

11. Pereira DCL, Lima SMRR. Prevalência de sobrepeso e obesidade em mulheres após a menopausa. Rev. eletrônica acervo saúde. 2018; 60(1): 1-6.

12. Agra G, Araújo LLLM, Pessoa VVB, Justino Filho J, Freire MEM, Formiga NS. Perception of obese women in relation their body. Revista Ibero-Americana de Saúde e Envelhecimento (Online). 2016 [Acesso em 15 agosto 2018]; 2017; 2(3): 775-93. Disponível em http://www.revistas.uevora.pt/index.php/saude envelhecimento/article/view/147/329

13. Poltronieri TS, Tusset C, Gregoletto MLO, Cremonese C. Insatisfação com a imagem corporal e fatores associados em mulheres do sul do Brasil. Rev. ciência \& saúde. 2016; 9(3): 128-34.

14. Silveira EA, Vieira LL, Souza JDD. Elevada prevalência de obesidade abdominal em idosos e associação com diabetes, hipertensão e doenças respiratórias. Ciênc. saúde coletiva (Online). 2018; 23(3): 903-12.

15. Ministério da Saúde (Br). Política Nacional de Alimentação e Nutrição. Brasília (DF): Ministério da Saúde; 2013.

16. Brischiliari SCR, Agnolo CMD, Gravena AAF, Lopes TCR, CarvaIho MDB, Pelloso SM. Doenças crônicas não transmissíveis e associação com fatores de risco. Rev. bras. cardiol. 2014; 27(1): 35-42. 17. Rouquayrol MZ, Gurgel MCS. Epidemiologia \& Saúde. 7ạ ed. Rio de Janeiro: Editora Med Book; 2013.

18. Castro JM, Ferreira EF, Silva DC, Oliveira RAR. Prevalence of overweight and obesity and the risk factors associated in adolescents. Rev. bras. obesidade, nutrição e emagrecimento. 2018; 12(69): 84-94.

19. Mais LA, Domene SMÁ, Barbosa MB, Taddei JADAC. Formação de hábitos alimentares e promoção da saúde e nutrição: o papel do nutricionista nos Núcleos de Apoio à Saúde da Família-NASF. Rev. APS. 2016; 18(2): 248-55.

20. Falkenberg MB, Mendes TDPL, Moraes EPD, Souza EMD. Educação em saúde e educação na saúde: conceitos e implicações para a saúde coletiva. Ciênc. saúde coletiva (Online) 2014; 19(3): 847-52. 21. Ferrari TK, Cesar CLG, Alves MCGP, Barros MBDA, Goldbaum M, Fisberg RM. Estilo de vida saudável em São Paulo, Brasil. Cad. Saúde Pública (Online). 2017; 33(1): e00188015.

22. Souza ACB, Oliveira JED, Caritá EC, Almeida CAN. Perfil dos pacientes obesos no primeiro atendimento em Ambulatório de Nutrologia Municipal de Ribeirão Preto (SP). Medicina (Ribeirão Preto). 2017; 50(4): 207-15.

23. Figueiredo MD, Cunha DR, Araujo AIG, Santos CM, Suplicy $\mathrm{HL}$, Boguszewski CL,et al.. Comportamento alimentar e perfil psicológico de mulheres obesas. Rev. PsicoFAE. 2014; 3(1): 43-54. 24. Oliveira V, Santos M, Andrade N, Teixeira C, Rodrigues F, Zanetti M. A percepção do corpo por mulheres com Diabetes Mellitus e obesidade. Rev. enferm. UERJ. 2014; 22(2): 251-7. 